\title{
Grothendieck Inclusion Systems
}

\author{
Răzvan Diaconescu
}

the date of receipt and acceptance should be inserted later

Keywords Grothendieck categories · inclusion systems · Grothendieck institutions

Abstract Inclusion systems have been introduced in algebraic specification theory as a categorical structure supporting the development of a general abstract logic-independent approach to the algebra of specification (or programming) modules. Here we extend the concept of indexed categories and their Grothendieck flattenings to inclusion systems. An important practical significance of the resulting Grothendieck inclusion systems is that they allow the development of module algebras for multi-logic heterogeneous specification frameworks. At another level, we show that several inclusion systems in use in some syntactic (signatures, deductive theories) or semantic contexts (models) appear as Grothendieck inclusion systems too. We also study several general properties of Grothendieck inclusion systems.

\section{Introduction}

Inclusion systems were introduced in [10] as a categorical device supporting an abstract general study of structuring of specification and programming modules that is independent of any underlying logic. There they were defined in a stronger version (corresponding to the epic inclusion systems with unions in our paper); here we use their weaker variant introduced by [4] under the name of 'weak inclusion systems'. Inclusion systems provide the underlying mathematical structure for module imports (which constitute the most fundamental structuring construct) in specification theory, and consequently have been used in a series of general module algebra studies such as $[10,14,8]$. Moreover they have also been used for developing axiomatizability $[23,7,8]$ and definability [1] results within the framework of the so-called 'institution-independent model theory' [8].

Inclusion systems capture categorically the concept of set-theoretic inclusion in a way reminiscent of how the rather notorious concept of factorization system [3] captures categorically the set-theoretic injections; however in many applications the former are more convenient than the latter. In fact, the applications to specification module algebra can be 
done only with inclusion systems, since factorization systems lack the uniqueness feature of inclusion systems.

Heterogeneous multi-logic specification has recently arisen as an important modern specification paradigm [25,9,22,21]. This involves specification frameworks based upon systems of logics rather than single logics. When the corresponding logics are formalized as 'institutions' in the sense of [12], one may homogenize the system of institutions to a single institution by a construction $[6,20]$ which extends the flattening of indexed categories defined by Alexandre Grothendieck within the context of algebraic geometry [15] to institutions. The great benefit of this flattening construction lies in the fact that the rather rich body of institution theoretic concepts, methods, and results can be applied directly to the resulting 'Grothendieck institution' instead of having to re-invent them from individual institutions to the significantly more sophisticated level of systems of institutions (as was done in [5]). In the case of inclusion systems the following important practical problem arises:

Assuming that each 'local' institution (of an indexed system of institutions) comes equipped with an inclusion system for its category of the signatures, do we have a canonical inclusion system for signatures of the corresponding 'global' Grothendieck institution?

In this paper we provide a definitive and complete answer to this problem by considering also an inclusion system for the indexation, i.e. for the category of the indices. In fact, this problem is only about the signatures of institutions, which means that in this case we have a problem only about categories equipped with inclusion systems, the concept of institution not being needed in this work. The canonicity of our Grothendieck inclusion system construction (Thm. 1) is expressed as an universal property (Prop. 3). Moreover, this construction applies also to contexts that are very different from the main motivation of our work, namely that of the inclusion systems for the signatures of Grothendieck institutions underlying multi-logic heterogeneous specification. These contexts are various examples of 'strong' inclusion systems in use in concrete specification frameworks Example 9), or from model theory (Example 10), or from abstract deductive theories (Example 11). Although each of these arise in a particular context, they have a common flavor (hence they are known in the literature as 'strong') which can be explained in the light of our work as being instances of a same general construction.

Our work also explores how some properties of inclusion systems which are important for the semantics of specification languages lift to Grothendieck inclusion systems.

The problem solved by our work has received preliminary answers in [5] and [6], the constructions proposed there can now be regarded as partial cases of the construction of Thm. 1.

The structure of the paper is as follows:

1. In the first section we recall the main concepts about inclusion systems. We also provide several important examples that will be used in the paper.

2. The next section is devoted to the Grothendieck construction on inclusion systems and to some of its general properties, including a universal caharcterization of this construction.

3. In the last technical section we sketch applications of Grothendieck inclusion systems to heterogeneous specification.

\section{Inclusion systems}

In this section we review briefly some basic concepts and facts regarding inclusion systems. This is organized as follows: 
1. We first recall some of the concepts and fix some notations from category theory needed by this work.

2. We recall the concept of inclusion system and present a series of examples.

3. We discuss some special properties of inclusion systems which are relevant for the theory of heterogeneous specification.

4. We organize inclusion systems as a 2-category and introduce a corresponding enriched concept of adjunction needed in this paper.

\subsection{Categorical preliminaries}

\subsubsection{Categories}

We assume the reader is familiar with basic notions and standard notations from category theory; e.g., see [17] for an introduction to this subject. Here we recall very briefly some of them. By way of notation, $|\mathbb{C}|$ denotes the class of objects of a category $\mathbb{C}, \mathbb{C}(A, B)$ the set of arrows with domain $A$ and codomain $B$, and composition is denoted by ";" and in diagrammatic order. The category of sets (as objects) and functions (as arrows) is denoted by $\operatorname{Set}$, and $\mathbb{C} a t$ is the category of all categories. ${ }^{1}$ The opposite of a category $\mathbb{C}$ (obtained by reversing the arrows of $\mathbb{C}$ ) is denoted $\mathbb{C}^{\mathrm{op}}$.

The application of functors $(\mathcal{U})$ (to either objects or arrows) can be also written in a diagrammatic way as $f \mathcal{U}$ rather than the more classical $\mathcal{U}(f)$. Sometimes it is even convenient to use subscripts or superscripts for the application of functors to objects or arrows.

\subsubsection{Grothendieck categories}

An indexed category is a functor $B: I^{\mathrm{op}} \rightarrow \mathbb{C}$ at; sometimes we denote $B(i)$ as $B_{i}$ (or $B^{i}$ ) for an index $i \in|I|$ and $B(u)$ as $B^{u}$ for an index morphism $u \in I$. Given an indexed category $B: I^{\mathrm{op}} \rightarrow \mathbb{C}$ at, let $B^{\sharp}$ be the Grothendieck category having $\langle i, \Sigma\rangle$, with $i \in|I|$ and $\Sigma \in\left|B^{i}\right|$, as objects and $\langle u, \varphi\rangle:\langle i, \Sigma\rangle \rightarrow\left\langle i^{\prime}, \Sigma^{\prime}\right\rangle$, with $u \in I\left(i, i^{\prime}\right)$ and $\varphi: \Sigma \rightarrow \Sigma^{\prime} B^{u}$, as arrows. The composition of arrows in $B^{\sharp}$ is defined by $\langle u, \varphi\rangle ;\left\langle u^{\prime}, \varphi^{\prime}\right\rangle=\left\langle u ; u^{\prime}, \varphi ; \varphi^{\prime} B^{u}\right\rangle$.

\subsubsection{2- and lax natural transformations}

Recall that a 2-natural transformation $\tau: F \Rightarrow G$ between 2-functors $F, G: \mathbb{A} \rightarrow \mathbb{B}$ maps any object $A$ of $|\mathbb{A}|$ to a 1 -cell $A \tau: F(A) \rightarrow G(A)$ such that $(A \tau) G(\sigma)=F(\sigma)(B \tau)$ for each 2-cell $\sigma: f \Rightarrow f^{\prime}: A \rightarrow B$.

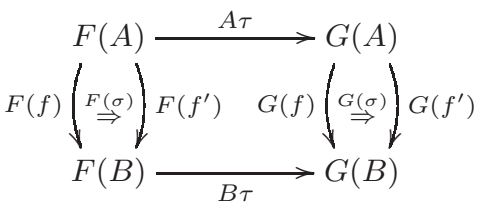

Lax natural transformations relax the commutativity of the natural transformation square above to the existence of 2-cells. Therefore a lax natural transformation $\tau$ between 2-functors $F$ and $G$ maps any object $A \in|\mathbb{A}|$ to $A \tau: F(A) \rightarrow G(A)$ and any 1-cell $u: A \rightarrow B$ to

\footnotetext{
1 Strictly speaking, this is only a quasi-category living in a higher set-theoretic universe.
} 
$u \tau: A \tau ; G(u) \Rightarrow F(u) ; B \tau$ such that $(F(\sigma)(B \tau)) ; f^{\prime} \tau=f \tau ;((A \tau) G(\sigma))$ for each 2-cell $\sigma: f \Rightarrow f^{\prime}: A \rightarrow B$ and

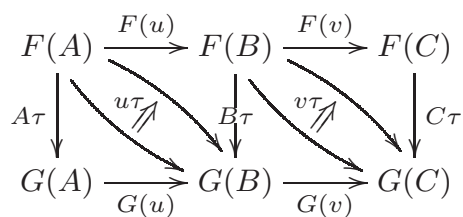

$(u ; v) \tau=(u \tau)(G(v)) ; F(u)(v \tau)$ for each $u: A \rightarrow B$ and $v: B \rightarrow C$.

2-categorical limits and co-limits are defined similarly to the conventional limits and co-limits as universal arrows from/to a diagonal functor. However, in the 2-categorical framework different concepts of natural transformations determine different concepts of (co-)limits. Therefore, when we employ 2-natural transformations we get the concepts of 2-(co-)limit as a final (initial) 2-(co-)cone, and when we employ lax natural transformations we get the concepts of lax (co-)limit as a final/initial lax cone/co-cone.

\subsection{Inclusion systems: definition and examples}

Definition 1 (Inclusion systems) $\langle\mathcal{I}, \mathcal{E}\rangle$ is a inclusion system for a category $\mathbb{C}$ if $\mathcal{I}$ and $\mathcal{E}$ are two sub-categories with $|\mathcal{I}|=|\mathcal{E}|=|\mathbb{C}|$ such that

1. $\mathcal{I}$ is a partial order (with the ordering relation denoted by $\subseteq$ ), and

2. every arrow $f$ in $\mathbb{C}$ can be factored uniquely as $f=e_{f} ; i_{f}$ with $e_{f} \in \mathcal{E}$ and $i_{f} \in \mathcal{I}$.

The arrows of $\mathcal{I}$ are called abstract inclusions, and the arrows of $\mathcal{E}$ are called abstract surjections. The domain of the inclusion $i_{f}$ in the factorization of $f$ is called the image of $f$ and is denoted as $\operatorname{Im}(f)$ or $f(A)$ when $A$ is a domain of $f$.

Note that there are no additional requirements such as the abstract surjections being epi or the abstract inclusions being mono. In [4] it is shown that the class $\mathcal{I}$ of the abstract inclusions and the class $\mathcal{E}$ of the abstract surjections determine each other. In this sense, [4] gives an explicit equivalent definition of inclusion systems which uses only the class $\mathcal{I}$ of the abstract inclusions.

Example 1 (Sets) The standard example of inclusion system is given by the category Set (with sets as objects and functions as arrows) which admits a non-trivial inclusion system where the abstract surjections are the surjective functions and the abstract inclusions are just the set inclusions.

Example 2 (Trivial inclusion systems) Any category admits a trivial inclusion system with any arrow being abstract surjection and the abstract inclusions being the identities.

Example 3 (Functor categories) For any inclusion system $\langle\mathcal{I}, \mathcal{E}\rangle$ and any category $\mathbb{J}$, the functor category $\langle\mathcal{I}, \mathcal{E}\rangle^{\mathbb{J}}$, i.e. the category that has the functors from $\mathbb{J}$ to the underlying category of $\langle\mathcal{I}, \mathcal{E}\rangle$ as objects and the natural transformations between these functors as arrows, admits an inclusion system such that a natural transformation is abstract inclusion or abstract surjection, respectively, if and only if all its components belong to $\mathcal{I}$ or $\mathcal{E}$, respectively.

The proof that this gives an inclusion system on $\langle\mathcal{I}, \mathcal{E}\rangle^{\mathbb{J}}$ uses the notorious Diagonal Fill-in Lemma (see [8]) and since it is rather straightforward it is left as exercise to the reader. 
Example 4 (Many-sorted signatures) A many-sorted (relational) signature is a pair $(S, P)$ consisting of

- a set of sort symbols $S$, and

- a family $P=\left\{P_{w} \mid w \in S^{*}\right\}$ of sets of relation (predicate) symbols indexed by arities.

Signature morphisms map the two components in a compatible way. This means that a signature morphism $\varphi:(S, P) \rightarrow\left(S^{\prime}, P^{\prime}\right)$ consists of

- a function $\varphi^{\text {st }}: S \rightarrow S^{\prime}$, and

- a family of functions $\varphi^{\mathrm{rl}}=\left\{\varphi_{w \rightarrow s}^{\mathrm{rl}}: P_{w} \rightarrow P_{\varphi^{\text {st }}(w)}^{\prime} \mid w \in S^{*}, s \in S\right\}$.

The concept of many-sorted signature presented above can be extended also with function symbols, arriving thus at the definition of many-sorted signature that is rather common in the practice of logic and specification. In fact, the existence of atoms and of (first-order) quantifications requires at least the presence of some constants (i.e. zero arity function symbols) in the signatures. However since our work does not involve sentences of many-sorted signatures, for the simplicity of our presentation, here we do not consider any function symbols. All results and concepts of this paper involving many-sorted signatures can be extended without any problem to the more refined concept of signature which considers functions symbols.

The category of many-sorted signatures admits the following non-trivial inclusion systems:

\begin{tabular}{l|l|l} 
inclusion system & $\begin{array}{l}\text { abstract surjections } \\
\varphi:(S, P) \rightarrow\left(S^{\prime}, P^{\prime}\right)\end{array}$ & $\begin{array}{l}\text { abstract inclusions } \\
(S, P) \hookrightarrow\left(S^{\prime}, P^{\prime}\right)\end{array}$ \\
\hline closed & $\varphi^{\text {st }}: S \rightarrow S^{\prime}$ surjective & $S \subseteq S^{\prime}$ \\
& & $P_{w}=P_{w}^{\prime}$ for $s \in S$ \\
\hline strong & $\varphi^{\text {st }}: S \rightarrow S^{\prime}$ surjective & $S \subseteq S^{\prime}$ \\
& $P_{w^{\prime}}^{\prime}=\bigcup_{\varphi^{\text {st }}}(w)=w^{\prime} \varphi^{\text {rl }}\left(P_{w}\right)$ & $P \subseteq \subseteq P_{w}^{\prime}$ for $s \in S$
\end{tabular}

A signature morphism $\varphi:(S, P) \rightarrow\left(S^{\prime}, P^{\prime}\right)$ preserves ad-hoc overloading ${ }^{2}$ when for each $\sigma \in P_{w} \cap P_{w^{\prime}}$ with $w$ and $w^{\prime}$ having the same length, we have that $\varphi_{w}(\sigma)=\varphi_{w^{\prime}}(\sigma)$. One may consider both the strong and the closed inclusion systems for the sub-category of the many-sorted signature morphisms preserving ad-hoc overloading.

Example 5 (Models) Models $M$ for a signature $(S, P)$ are structures interpreting each sort symbol $s$ as a set $M_{s}$, and each relation symbol $\pi$ as a subset $M_{\pi}$ of $M_{w}$, the product of the interpretations of the argument sorts. A model homomorphism $h: M \rightarrow M^{\prime}$ is an indexed family of functions $\left\{h_{s}: M_{s} \rightarrow M_{s}^{\prime}\right\}_{s \in S}$ such that

- $h_{w}(m) \in M_{\pi}^{\prime}$ if $m \in M_{\pi}$ (i.e. $h_{w}\left(M_{\pi}\right) \subseteq M_{\pi}^{\prime}$ ) for each relation $\pi \in P_{w}$ and each $m \in M_{w}$.

where $h_{w}: M_{w} \rightarrow M_{w}^{\prime}$ is the canonical component-wise extension of $h$, i.e. $h_{w}\left(m_{1} \ldots m_{n}\right)=$ $h_{s_{1}}\left(m_{1}\right) \ldots h_{s_{n}}\left(m_{n}\right)$ for $w=s_{1} \ldots s_{n}$ and $m_{i} \in M_{s_{i}}$.

A model homomorphism $h: M \rightarrow N$

- is closed when $M_{\pi}=h_{w}^{-1}\left(N_{\pi}\right)$ for each relation symbol $\pi \in P_{w}$, and

- is strong when $h_{w}\left(M_{\pi}\right)=N_{\pi}$ for each relation symbol $\pi \in P_{w}$.

\footnotetext{
2 Terminology due to Till Mossakowski.
} 
For each model homomorphism $M \rightarrow N$ which is a set inclusion for each sort $s \in S$ let us say that $M$ is a submodel of $N$.

For any signature $(S, P)$, the category of $(S, P)$-models admits the following two inclusion systems:

\begin{tabular}{l|l|l} 
inclusion system & abstract surjections & abstract inclusions \\
\hline closed & surjective homomorphisms & closed sub-models \\
\hline strong & strong surjective homomorphisms & sub-models
\end{tabular}

Example 6 (Theories) Following the work of Fiadeiro and Sernadas [11], logical systems based on deduction can be formalized as $\pi$-institutions, which have for each signature $\Sigma$ a set of $\Sigma$-sentences, but no given models. To compensate for this lack, a consequence relation is given on sentences. We will use the definition of Fiadeiro and Sernadas [11] as modified by Meseguer [19], rather than that of Maibaum and Fiadeiro [18]; Harper, Sannella and Tarlecki [16] have given a definition similar to Meseguer's, but restricted to finite sets of sentences. Thus a $\pi$-institution consists of

1. a category $\mathbb{S i g}$, whose objects are called signatures,

2. a functor Sen: $\mathbb{S i g} \rightarrow$ Set, giving for each signature a set whose elements are called sentences over that signature, and

3. a relation $\vdash_{\Sigma} \subseteq \mathcal{P}(\operatorname{Sen}(\Sigma)) \times \operatorname{Sen}(\Sigma)$ for each $\Sigma \in \mid$ Sig $\mid$, called $\Sigma$-consequence, ${ }^{3}$

such that the following conditions hold:

reflexivity $\{e\} \vdash_{\Sigma} e$ for each $e \in \operatorname{Sen}(\Sigma)$;

monotonicity if $E \vdash_{\Sigma} e$ and $E \subseteq E^{\prime}$ then $E^{\prime} \vdash_{\Sigma} e$;

transitivity if $E \vdash_{\Sigma} e^{\prime}$ for each $e^{\prime} \in E^{\prime}$ and if $\left(E \cup E^{\prime}\right) \vdash_{\Sigma} e$, then $E \vdash_{\Sigma} e$;

translation if $E \vdash_{\Sigma} e$ and if $\varphi: \Sigma \rightarrow \Sigma^{\prime}$ in $\operatorname{Sig}$, then $\operatorname{Sen}(\varphi)(E) \vdash_{\Sigma^{\prime}} \operatorname{Sen}(\varphi)(e)$.

In general we prefer to write $\varphi(e)$ instead of $\operatorname{Sen}(\varphi)(e)$.

The 'institutions' of Goguen and Burstall [12] appear canonically as a $\pi$-institutions by considering the semantic consequence relations $\models_{\Sigma}$ in the role of the consequence relations $\vdash_{\Sigma}$. Thus one can say $\pi$-institutions are more abstract than institutions. However, each $\pi$ institution can be given a rather artificial model theory by a comma category construction on theories [19].

Theories represent one of the two major ways to provide denotations for software modules or structured specifications, which has been exploited in works such as $[2,10,14]$. The other more subtle semantic way, which requires the framework of institutions, is that of [24], employed also by [8], in which denotations of specifications are classes of models. In any $\pi$-institution, a theory $(\Sigma, E)$, also called $\Sigma$-theory, consists of a signature $\Sigma$ and a set $E$ of $\Sigma$-sentences closed under consequence, i.e. if $E \vdash_{\Sigma} e$ then $e \in E$. A theory $(\Sigma, E)$ is presented by a set $E_{0}$ of sentences when $E_{0} \subseteq E$ and $E_{0} \vdash_{\Sigma} E$ (meaning that $E_{0} \vdash_{\Sigma} e$ for each $e \in E)$. This is denoted by $E=E_{0}^{\bullet}$. A theory morphism $\varphi:(\Sigma, E) \rightarrow\left(\Sigma^{\prime}, E^{\prime}\right)$ is just a signature morphism $\varphi: \Sigma \rightarrow \Sigma^{\prime}$ such that $\varphi(E) \subseteq E^{\prime}$. Note that theory morphisms form a category under the composition given by the composition of the signature morphisms. A theory morphism $\varphi:(\Sigma, E) \rightarrow\left(\Sigma^{\prime}, E^{\prime}\right)$

- is closed when $E=\varphi^{-1}\left(E^{\prime}\right)$, and

- is strong when $E^{\prime}=\varphi(E)^{\bullet}$.

\footnotetext{
${ }^{3}$ Here $\mathcal{P}$ denotes the power set function.
} 
Any inclusion system $\langle\mathcal{I}, \mathcal{E}\rangle$ for the category $\mathbb{S} i g$ of the signatures determines two inclusion systems for the category of theories as follows:

\begin{tabular}{l|l|l} 
inclusion system & abstract surjections $\varphi$ & abstract inclusions $\varphi$ \\
\hline closed & $\varphi \in \mathcal{E}$ & $\varphi \in \mathcal{I}$ and $\varphi$ closed theory morphism \\
\hline strong & $\varphi \in \mathcal{E}$ and $\varphi$ strong theory morphism & $\varphi \in \mathcal{I}$
\end{tabular}

\subsection{Special properties of inclusion systems}

The abstract surjections of some inclusion systems need not necessarily be surjective in the ordinary set-theoretic sense. However some results involving inclusion systems requires the epi property for the abstract surjections.

Definition 2 (Epic inclusion systems) An inclusion system is epic when all abstract surjections are epis.

Example 7 The standard inclusion system of Set is epic, while the trivial one for the same category Set is not. The strong inclusion systems for the many sorted signatures is epic (for signature morphisms preserving ad-hoc overloading see Cor. 4 below) while the closed one is not. Both the strong and the closed inclusion systems for models of many-sorted signatures are epic (for the strong one see also Cor. 5). Both the strong and the closed inclusion systems for theories are epic when the underlying inclusion system of signatures is epic (for the strong one see also Cor. 6 below).

The concept below is critical for the semantics of (software) module imports and is one of the important features that distinguishes inclusion systems from factorization systems in the sense that the latter can not support such concept in a proper way.

Definition 3 (Unions) An inclusion system $\langle\mathcal{I}, \mathcal{E}\rangle$ has unions when $\mathcal{I}$ has finite least upper bounds (denoted $\cup$ ).

Example 8 The standard inclusion system of Set has unions which are exactly the usual unions of sets, while the trivial inclusion system of Set evidently does not have unions. The strong inclusion systems for many-sorted signatures and for the models have unions (for signature morphisms preserving ad-hoc overloading see Cor. 1 and for models see Cor. 2 , respectively), while the closed ones for the same categories do not have unions. The strong inclusion system for theories has unions when the underlying inclusion systems for the signatures has unions (see Cor. 3 below). In general the closed inclusion system for theories does not have unions.

\subsection{The category of inclusion systems}

The definition below gives the concept of (homo)morphism of inclusion systems.

Definition 4 (Inclusive functors) A functor $\mathcal{U}:\langle\mathcal{I}, \mathcal{E}\rangle \rightarrow\left\langle\mathcal{I}^{\prime}, \mathcal{E}^{\prime}\right\rangle$ (between the underlying categories of the inclusion systems) is inclusive when it preserves the inclusions, i.e. $\mathcal{U}(\mathcal{I}) \subseteq$ $\mathcal{I}^{\prime}$. 
One may wonder whether inclusive functors is the right concept of morphism of inclusion systems since it does not consider directly the abstract surjections. The answer to this is given by the fact, already mentioned above, that the class of the abstract inclusions and the class of the abstract surjections of an inclusion system determine each other [4], and consequently it is possible to have an equivalent definition, even if not as intuitive as the current one, of inclusion systems only in terms of the class of the abstract inclusions.

Fact 21 Inclusion systems and inclusive functors form a category denoted $\mathbb{I} \mathbb{S}$. Moreover, IS can be endowed with a 2-categorical structure with the 2-cells being defined as natural transformations between inclusive functors such that all their components are inclusions.

The equational definition of the concept of adjunction by the so-called 'triangular laws' (see [17]) permits the well known generalization of the concept of adjunction from $\mathbb{C} a t$ to abstract 2-categories. Let us call adjunctions defined in $\mathbb{I S}$ as $\mathbb{I S}$-adjunctions.

Fact 22 An IS-adjunction between inclusion systems consists of an adjunction between the underlying categories such that both the left and the right adjoints are inclusive functors and such that all components of the unit and the co-unit are inclusions.

\section{Grothendieck inclusion systems}

This is the main technical part of the paper. It is organized as follows:

1. We develop the general Grothendieck construction on inclusion systems.

2. We show how the examples of strong inclusion systems presented in the Sect. 2.2 arise as instances of the general Grothendieck inclusion systems.

3. We develop a general result on existence of unions for Grothendieck inclusion systems and apply this to our benchmark examples.

4. We develop a general result on Grothendieck inclusion systems being epic, and apply this to our benchmark examples.

5. We characterize Grothendieck inclusion systems by the universal property of an enriched colimit.

6. The examples of closed inclusion systems presented in Sect. 2.2 are captured by a general inclusion system construction on the Grothendieck categories resulting from categories indexed by inclusion systems. However this construction is rather gross since it does not consider any inclusion system structure on the 'local' categories, hence it is inappropriate for the semantics of structured heterogeneous specifications.

\subsection{The basic construction}

The following definition extends the concept of indexed category, first by considering inclusion systems instead of categories, and second by considering a structure of inclusion system on the category of the indices.

Definition 5 An enriched indexed inclusion system is a functor $B:\langle\mathcal{I}, \mathcal{E}\rangle \rightarrow \mathbb{I} \mathbb{S}^{\text {op }}$ from the underlying category of an inclusion system 'of indices' to the opposite of the category of inclusions systems and inclusive functors. 
Definition 6 An enriched indexed inclusion system $B:\langle\mathcal{I}, \mathcal{E}\rangle \rightarrow \mathbb{I} \mathbb{S}^{\text {op }}$ is invertible when for each index morphism $u$, the corresponding inclusive functor $B^{u}$ has an ISS-left-adjoint denoted $[-]^{u}$. It is $\mathcal{E}$-invertible when the $\mathbb{I S}$-left-adjoint to $B^{u}$ exists for $u \in \mathcal{E}$ (and not necessarily for all index morphisms $u$ ).

The following is the main result of this paper.

Theorem 1 For any $\mathcal{E}$-invertible enriched indexed inclusion system $B:\langle\mathcal{I}, \mathcal{E}\rangle \rightarrow \mathbb{I} \mathbb{S}^{\text {op }}$ the Grothendieck category $B^{\sharp}$ of $B^{\mathrm{op}} ;(\mathbb{I S} \rightarrow \mathbb{C}$ at) (from the opposite of the underlying category of $\langle\mathcal{I}, \mathcal{E}\rangle$ to $\mathbb{C}$ at) can be endowed with an inclusion system $\left\langle\mathcal{I}^{\sharp}, \mathcal{E}^{\sharp}\right\rangle$ such that $\langle u, \varphi\rangle:\langle j, \Sigma\rangle \rightarrow\left\langle j^{\prime}, \Sigma^{\prime}\right\rangle$ is

- an abstract inclusion iff both $u$ and $\varphi$ are abstract inclusions, and

- an abstract surjection iff $u$ is an abstract surjection and $\Sigma^{\prime}=[\varphi(\Sigma)]^{u}$.

Proof For each index $j$, let $B^{j}=\left\langle\mathcal{I}^{j}, \mathcal{E}^{j}\right\rangle$ be the inclusion system corresponding to the index $j$. For each index morphism $u: j \rightarrow j^{\prime}$ let $\eta^{u}$ and $\varepsilon^{u}$, respectively, be the unit and the co-unit, respectively, of the corresponding adjunction (with $B^{u}$ the $\mathbb{I S}$-right-adjoint and $[-]^{u}$ the ISS-left-adjoint).

First we show that $\mathcal{I}^{\sharp}$ is a sub-category of $B^{\sharp}$. Let $\langle u, \varphi\rangle$ and $\left\langle u^{\prime}, \varphi^{\prime}\right\rangle$ be composable abstract inclusions. Then $u ; u^{\prime}$ is an abstract inclusions of indices. Because $\varphi^{\prime} \in \mathcal{I}^{j^{\prime}}$ and $B^{u}$ is inclusive, we have that $\varphi^{\prime} B^{u} \in \mathcal{I}^{j}$. Since $\varphi \in \mathcal{I}^{j}$ we have that $\varphi ; \varphi^{\prime} B^{u} \in \mathcal{I}^{j}$. Hence $\langle u, \varphi\rangle ;\left\langle u^{\prime}, \varphi^{\prime}\right\rangle=\left\langle u ; u^{\prime}, \varphi ; \varphi^{\prime} B^{u}\right\rangle \in \mathcal{I}^{\sharp}$.

For showing that $\mathcal{I}^{\sharp}$ is a poset let $\left\langle u_{1}, \varphi_{1}\right\rangle,\left\langle u_{2}, \varphi_{2}\right\rangle:\langle j, \Sigma\rangle \rightarrow\left\langle j^{\prime}, \Sigma^{\prime}\right\rangle$ be a abstract inclusions. Because $u_{1}, u_{2}: j \rightarrow j^{\prime}$ are abstract inclusions, we have that $u_{1}=u_{2}$. Thus $\varphi_{1}, \varphi_{2}: \Sigma \rightarrow \Sigma B^{u_{i}}$ are abstract inclusions, hence $\varphi_{1}=\varphi_{2}$.

In order to show that $\mathcal{E}^{\sharp}$ is a sub-category, consider abstract surjections $\langle u, \varphi\rangle:\langle j, \Sigma\rangle \rightarrow$ $\left\langle j^{\prime}, \Sigma^{\prime}\right\rangle$ and $\left\langle u^{\prime}, \varphi^{\prime}\right\rangle:\left\langle j^{\prime}, \Sigma^{\prime}\right\rangle \rightarrow\left\langle j^{\prime \prime}, \Sigma^{\prime \prime}\right\rangle$. Obviously $u ; u^{\prime}$ is an abstract surjection of indices and we have to show that $\Sigma^{\prime \prime}=\left[\left(\varphi ; \varphi^{\prime} B^{u}\right)(\Sigma)\right]^{u ; u^{\prime}}$.

Let us factor $\varphi=e_{\varphi} ; i_{\varphi}$ such that $e_{\varphi} \in \mathcal{E}^{j}$ and $i_{\varphi} \in \mathcal{I}^{j}$ and $\varphi^{\prime}=e_{\varphi^{\prime}} ; i_{\varphi^{\prime}}$ such that $e_{\varphi^{\prime}} \in \mathcal{E}^{j^{\prime}}$ and $i_{\varphi^{\prime}} \in \mathcal{I}^{j^{\prime}}$. Because $\langle u, \varphi\rangle$ and $\left\langle u^{\prime}, \varphi^{\prime}\right\rangle$ are abstract surjections we have that $\Sigma^{\prime}=[\varphi(\Sigma)]^{u}$ and $\Sigma^{\prime \prime}=\left[\varphi^{\prime}\left(\Sigma^{\prime}\right)\right]^{u^{\prime}}$ and that $i_{\varphi}$ and $i_{\varphi^{\prime}}$, respectively, are precisely the universal arrows $\eta_{\varphi(\Sigma)}^{u}$ and $\eta_{\varphi^{\prime}\left(\Sigma^{\prime}\right)}^{u^{\prime}}$, respectively.

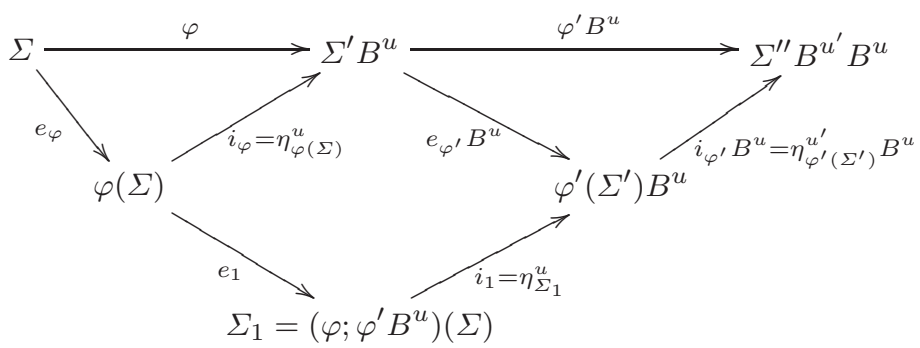

Let us also factor $\eta_{\varphi(\Sigma)}^{u} ; e_{\varphi^{\prime}} B^{u}=e_{1} ; i_{1}$ such that $\left(e_{1}: \varphi(\Sigma) \rightarrow \Sigma_{1}\right) \in \mathcal{E}^{j}$ and $\left(i_{1}: \Sigma_{1} \rightarrow\right.$ $\left.\varphi^{\prime}\left(\Sigma^{\prime}\right) B^{u}\right) \in \mathcal{I}^{j}$. Since $B^{u}$ is inclusive, we have that $\eta_{\varphi^{\prime}\left(\Sigma^{\prime}\right)}^{u^{\prime}} B^{u} \in \mathcal{I}^{j}$, hence $\Sigma_{1}$ is the image of $\varphi ; \varphi^{\prime} B^{u}$. Because $\left(i_{1}: \Sigma_{1} \rightarrow \varphi^{\prime}\left(\Sigma^{\prime}\right) B^{u}\right) \in \mathcal{I}^{j}$ and because $[-]^{u}$ is inclusive we have that $\left(\left[i_{1}\right]^{u}:\left[\Sigma_{1}\right]^{u} \rightarrow\left[\varphi^{\prime}\left(\Sigma^{\prime}\right) B^{u}\right]^{u}\right) \in \mathcal{I}^{j^{\prime}}$. Since the co-unit component $\varepsilon_{\varphi^{\prime}\left(\Sigma^{\prime}\right)}^{u} \in$ $\mathcal{I}^{j^{\prime}}$ we have that $\left(\left[i_{1}\right]^{u} ; \varepsilon_{\varphi^{\prime}\left(\Sigma^{\prime}\right)}^{u}:\left[\Sigma_{1}\right]^{u} \rightarrow \varphi^{\prime}\left(\Sigma^{\prime}\right)\right) \in \mathcal{I}^{j^{\prime}}$. From this we now obtain the following: 


$$
\begin{aligned}
{\left[e_{1}\right]^{u} ;\left(\left[i_{1}\right]^{u} ; \varepsilon_{\varphi^{\prime}\left(\Sigma^{\prime}\right)}^{u}\right) } & =\left[\eta_{\varphi(\Sigma)}^{u}\right]^{u} ;\left[e_{\varphi^{\prime}} B^{u}\right]^{u} ; \varepsilon_{\varphi^{\prime}\left(\Sigma^{\prime}\right)}^{u} & & \\
& =\left[\eta_{\varphi(\Sigma)}^{u}\right]^{u} ; \varepsilon_{\Sigma^{\prime}}^{u} ; e_{\varphi^{\prime}} & & \text { by the naturality of } \varepsilon^{u} \\
& =e_{\varphi^{\prime}} & & \text { by the triangular laws. }
\end{aligned}
$$

By the uniqueness of the factorization we deduce that $\left[i_{1}\right]^{u} ; \varepsilon_{\varphi^{\prime}\left(\Sigma^{\prime}\right)}^{u}$ is identity, hence $\left[\Sigma_{1}\right]^{u}=$ $\varphi^{\prime}\left(\Sigma^{\prime}\right)$ and thus $i_{1}$ is the unit component $\eta_{\Sigma_{1}}^{u}$. We may finish this argument by noticing that $\eta_{\Sigma_{1}}^{u} ; \eta_{\varphi^{\prime}\left(\Sigma^{\prime}\right)}^{u^{\prime}} B^{u}$ being both universal arrow and inclusion, it should be in fact the unit component $\eta_{\Sigma_{1}}^{u ; u^{\prime}}$, hence $\Sigma^{\prime \prime}=\left[\left(\varphi ; \varphi^{\prime} B^{u}\right)(\Sigma)\right]^{u ; u^{\prime}}$.

We now consider an arrow $\langle u, \varphi\rangle:\langle j, \Sigma\rangle \rightarrow\left\langle j^{\prime}, \Sigma^{\prime}\right\rangle$ in the Grothendieck category $B^{\sharp}$ and prove that it factors uniquely as a composition between an arrow from $\mathcal{E}^{\sharp}$ and an arrow from $\mathcal{I}^{\sharp}$. We factor

- $u=e_{u} ; i_{u}$ such that $e_{u} \in \mathcal{E}$ and $i_{u} \in \mathcal{I}$, and

- $\varphi=e_{\varphi} ; i_{\varphi}$ such that $e_{\varphi} \in \mathcal{E}^{j}$ and $i_{\varphi} \in \mathcal{I}^{j}$.
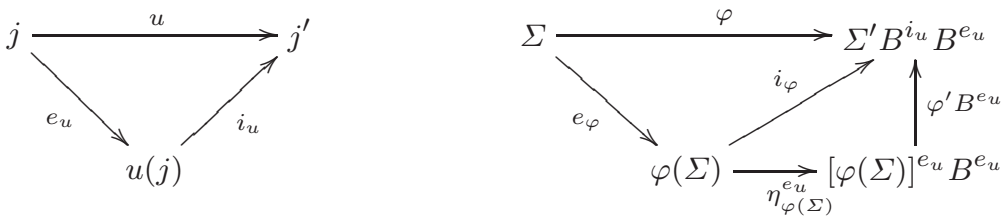

Because $\varphi(\Sigma) \subseteq \Sigma^{\prime} B^{i_{u}} B^{e_{u}}$, by an argument similar to the proof of $\left[\Sigma_{1}\right]^{u} \subseteq \varphi^{\prime}\left(\Sigma^{\prime}\right)$, we have that $[\varphi(\Sigma)]^{e_{u}} \subseteq \Sigma^{\prime} B^{i_{u}}$. Let us denote this inclusion by $\varphi^{\prime}$. Then $\langle u, \varphi\rangle$ factors as $\left\langle e_{u}, e_{\varphi} ; \eta_{\varphi(\Sigma)}^{e_{u}}\right\rangle ;\left\langle i_{u}, \varphi^{\prime}\right\rangle$. Obviously $\left\langle i_{u}, \varphi^{\prime}\right\rangle$ is an abstract inclusion. In order to establish that the arrow $\left\langle e_{u}, e_{\varphi} ; \eta_{\varphi(\Sigma)}^{e_{u}}\right\rangle:\langle j, \Sigma\rangle \rightarrow\left\langle u(j),[\varphi(\Sigma)]^{e_{u}}\right\rangle$ is an abstract surjection we need to check the equality $[\varphi(\Sigma)]^{e_{u}}=\left[\left(e_{\varphi} ; \eta_{\varphi(\Sigma)}^{e_{u}}\right)(\Sigma)\right]^{e_{u}}$. This holds because $\operatorname{Im}\left(e_{\varphi} ; \eta_{\varphi(\Sigma)}^{e_{u}}\right)=$ $\varphi(\Sigma)$ (since $e_{\varphi}$ is abstract surjection and $\eta_{\varphi(\Sigma)}^{e_{u}}$ is abstract inclusion as a unit component).

The uniqueness of this factorization follows stepwise from the uniqueness of the factorization of the index morphism, then from the uniqueness of the factorization through the inclusion system $\left\langle\mathcal{I}^{j}, \mathcal{E}^{j}\right\rangle$, and finally from the uniqueness of $\varphi(\Sigma)^{e_{u}}$ as a free object. Let us omit here the details of this proof.

\subsection{Examples}

\section{Example 9 (Many-sorted signatures) Let}

- $\langle\mathcal{I}, \mathcal{E}\rangle$ be the standard inclusion system on $\mathbb{S e t}$, the category of sets (cf. Example 1).

- $B:\langle\mathcal{I}, \mathcal{E}\rangle \rightarrow \mathbb{I} \mathbb{S}^{\text {op }}$ be the functor such that

- $B$ maps each set $S$ to the category $B(S)$ whose objects are families of sets $P=$ $\left\{P_{w} \mid w \in S^{*}\right\}$. Arrows between $P$ and $P^{\prime}$ are families of functions $\left\{f_{w}: P_{w} \rightarrow\right.$ $\left.P_{w}^{\prime} \mid w \in S^{*}\right\}$. The abstract inclusions and the abstract surjections, respectively, are point-wise inclusions and surjections, respectively, i.e. for each $w \in S^{*}$ the function $f_{w}$ is inclusion or surjection, respectively. 
- $B$ maps each function $u: S \rightarrow S^{\prime}$ to the functor $B^{u}: B\left(S^{\prime}\right) \rightarrow B(S)$ defined by $\left(B^{u}\left(P^{\prime}\right)\right)_{w}=P_{u(w)}^{\prime}$ for each $P^{\prime} \in B\left(S^{\prime}\right)$ and each $w \in S^{*}$. Each $B^{u}$ has a leftadjoint $[-]^{u}$ such that $[P]_{w^{\prime}}^{u}=\biguplus_{u(w)=w^{\prime}} P_{w}$ for any $P \in|B(S)|$ and any $w^{\prime} \in S^{\prime^{*}}$ (where $\uplus$ denotes the disjoint union).

Unfortunately in general $[-]^{u}$ is not an $\mathbb{I S}$-left-adjoint, hence the strong inclusion system for many-sorted signatures does not arise as an example of Thm. 1! However this situation can be saved if we considered only the signature morphisms that preserve ad-hoc overloading, in that case $[P]_{w^{\prime}}^{u}$ being $\bigcup_{u(w)=w^{\prime}} P_{w}$. Therefore if in $B(S)$ we allow only arrows $P \rightarrow P^{\prime}$ that preserves ad-hoc overloading (see Ex. 4), the Grothendieck inclusion system arising from the application of Thm. 1 is precisely the strong inclusion system for the signature morphisms preserving ad-hoc overloading.

Example 10 (Models) Given a signature $(S, P)$, the strong inclusion system for its category of models is the Grothendieck inclusion system $\left\langle\mathcal{I}^{\sharp}, \mathcal{E}^{\sharp}\right\rangle$ of Thm. 1 where $B:\langle\mathcal{I}, \mathcal{E}\rangle \rightarrow$ $\mathbb{I} \mathbb{S}^{\mathrm{OP}}$ is the invertible enriched indexed inclusion system defined as follows:

$-\langle\mathcal{I}, \mathcal{E}\rangle$ is the standard inclusion system of the (functor) category $\operatorname{Set}^{S}$ of $S$-sorted sets, where for each $S$-sorted sets $M$ and $N, h=\left\{h_{s}: M_{s} \rightarrow N_{s} \mid s \in S\right\}$ is an abstract inclusion if and only if $h_{s}$ is an inclusion $M_{s} \subseteq N_{s}$ for each $s \in S$, and is an abstract surjection if and only if $h_{s}$ is surjective function for each $s \in S$.

- $B$ maps each $S$-sorted set $M$ the poset $B(\Sigma)$ of the $(S, P)$-models $M^{\prime}$ such that $M_{s}^{\prime}=$ $M_{s}$ for each $s \in S$. Then $M^{\prime} \leq M^{\prime \prime}$ when $M_{\pi}^{\prime} \subseteq M_{\pi}^{\prime \prime}$ for each $\pi \in P$. B( $\left.\Sigma\right)$ is considered with the standard inclusion system for posets (in which the abstract surjections are the identities).

- $B$ maps each $S$-sorted function $h: M \rightarrow N$ to the monotonic function $B^{h}: B(N) \rightarrow$ $B(M)$ defined for each $N^{\prime} \in B(N)$ by $\left(B^{h}\left(N^{\prime}\right)\right)_{\pi}=h_{w}^{-1}\left(N_{\pi}^{\prime}\right)$ for each $\pi \in P_{w}$.

For each $S$-sorted function $h: M \rightarrow N$, the functor $B^{h}$ has a left adjoint [-] $]^{h}$ such that for each $M^{\prime} \in B(M),\left(\left[M^{\prime}\right]^{h}\right)_{\pi}=h_{w}\left(M_{\pi}^{\prime}\right)$ for each $\pi \in P_{w}$. The resulting adjunction is trivially an $\mathbb{I S}$-adjunction since all arrows of $B(M)$ 's are inclusions.

Example 11 (Theories) Given a $\pi$-institution $(\mathbb{S i g}$, Sen, $\vdash$ ) with an inclusion system $\langle\mathcal{I}, \mathcal{E}\rangle$ for the category $\mathbb{S i g}$ of the signatures, the strong inclusion system for its category of theories is the Grothendieck inclusion system $\left\langle\mathcal{I}^{\sharp}, \mathcal{E}^{\sharp}\right\rangle$ of Thm. 1 where $B:\langle\mathcal{I}, \mathcal{E}\rangle \rightarrow \mathbb{I} \mathbb{S}^{\text {op }}$ is the invertible enriched indexed inclusion system defined as follows:

- $B$ maps each signature $\Sigma$ to the poset (under set inclusion) $B(\Sigma)$ of the $\Sigma$-theories, which is considered with its standard inclusion system (in which the abstract surjections are the identities) and

- $B$ maps each signature morphism $\varphi: \Sigma \rightarrow \Sigma^{\prime}$ to the monotonic function $B^{\varphi}: B\left(\Sigma^{\prime}\right) \rightarrow$ $B(\Sigma)$ defined by $B^{\varphi}\left(\Sigma^{\prime}, E^{\prime}\right)=\left(\Sigma, \varphi^{-1}\left(E^{\prime}\right)\right)$ for each $E^{\prime} \subseteq \operatorname{Sen}\left(\Sigma^{\prime}\right)$ such that $\left(\Sigma^{\prime}, E^{\prime}\right)$ is theory. (Note that in this case $\left(\Sigma, \varphi^{-1}\left(E^{\prime}\right)\right)$ is a theory too.)

For each signature morphism $\varphi: \Sigma \rightarrow \Sigma^{\prime}$ the functor $B^{\varphi}$ has a left adjoint $[-]^{\varphi}$ such that $[E]^{\varphi}=\varphi(E)^{\bullet}$. The resulting adjunction is trivially an $\mathbb{I S}$-adjunction since all arrows of $B(\Sigma)$ 's are inclusions.

\subsection{Unions}

The following result develops sufficient conditions for unions for Grothendieck inclusion systems and requires invertible enriched indexed inclusion systems which is stronger than the $\mathcal{E}$-invertible condition of Thm. 1 . 
Proposition 1 For any invertible enriched indexed inclusion system $B:\langle\mathcal{I}, \mathcal{E}\rangle \rightarrow \mathbb{I} \mathbb{S}^{o p}$, the Grothendieck inclusion system $\left\langle\mathcal{I}^{\sharp}, \mathcal{E}^{\sharp}\right\rangle$ has unions if

- the inclusion system of indices $\langle\mathcal{I}, \mathcal{E}\rangle$ has unions, and

- for each index $j$ the 'local' inclusion system $B^{j}=\left\langle\mathcal{I}^{j}, \mathcal{E}^{j}\right\rangle$ has unions.

Proof We prove that $\left\langle j_{1}, \Sigma_{1}\right\rangle \cup\left\langle j_{2}, \Sigma_{2}\right\rangle=\left\langle j_{1} \cup j_{2},\left[\Sigma_{1}\right]^{u_{1}} \cup\left[\Sigma_{2}\right]^{u_{2}}\right\rangle$ where $u_{1}: j_{1} \rightarrow$ $j_{1} \cup j_{2}$ and $u_{2}: j_{2} \rightarrow j_{1} \cup j_{2}$ are the inclusions to the union.

Assume $\left\langle j_{1}, \Sigma_{1}\right\rangle \subseteq\langle j, \Sigma\rangle$ and $\left\langle j_{2}, \Sigma_{2}\right\rangle \subseteq\langle j, \Sigma\rangle$. We have that $j_{1} \cup j_{2} \subseteq j$, we denote this inclusion by $u$. Because $\Sigma_{1} \subseteq \Sigma B^{u_{1} ; u}=\Sigma B^{u} B^{u_{1}}$ we have that $\left[\Sigma_{1}\right]^{u_{1}} \subseteq \Sigma B^{u}$. Similarly $\left[\Sigma_{2}\right]^{u_{2}} \subseteq \Sigma B^{u}$. This shows that $\left\langle j_{1} \cup j_{2},\left[\Sigma_{1}\right]^{u_{1}} \cup\left[\Sigma_{2}\right]^{u_{2}}\right\rangle \subseteq\langle j, \Sigma\rangle$.

Corollary 1 The strong inclusion system of many-sorted signature morphisms preserving ad-hoc overloading has unions.

Proof We check the conditions of Prop. 1.

- The corresponding enriched inclusion system $B$ is invertible since for each function $f: S \rightarrow S^{\prime}, B^{f}$ has an $\mathbb{I} S$-left-adjoint (cf. Example 9).

- The standard inclusion system of $\mathbb{S e t}$ has unions, which are the usual set unions.

- For each set $S, B(S)$ has point-wise unions defined by

$$
\left(P \cup P^{\prime}\right)_{w}=P_{w} \cup P_{w}^{\prime} .
$$

The inclusion system of all many-sorted signatures also has unions, but this result cannot be obtained by means of Prop. 1.

Corollary 2 For each many-sorted signature $(S, P)$, the strong inclusion system of $(S, P)$ models has unions.

Proof We check the conditions of Prop. 1.

- The corresponding enriched inclusion system $B$ is invertible since for each $S$-sorted function $h: M \rightarrow N, B^{h}$ has an IS-left-adjoint (cf. Example 10).

- The standard inclusion system of Set $^{S}$, the category of $S$-sorted sets, has 'point-wise' unions.

- For each $S$-sorted set $M, B(M)$ has unions defined by

$$
\left(M^{\prime} \cup M^{\prime \prime}\right)_{\pi}=M_{\pi}^{\prime} \cup M_{\pi}^{\prime \prime}
$$

Corollary 3 For any $\pi$-institution ( ig, Sen, $\vdash$ ), the strong inclusion system of theories has unions when the underlying inclusion system for $\mathbb{S} i g$ has unions.

Proof We check the other two conditions of Prop. 1 besides that mentioned in the statement of the corollary.

- The corresponding enriched inclusion system $B$ is invertible, cf. Example 11.

- For each signature $\Sigma, B(\Sigma)$ has unions defined by

$$
(\Sigma, E) \cup\left(\Sigma, E^{\prime}\right)=\left(\Sigma,\left(E \cup E^{\prime}\right)^{\bullet}\right) .
$$


3.4 Epic

The following result develops sufficient conditions for the Grothendieck inclusion systems to be epic.

Proposition 2 In addition to the conditions of Thm. 1 if the inclusion system of the indices $\langle\mathcal{I}, \mathcal{E}\rangle$ is epic, $B^{j}=\left\langle\mathcal{I}^{j}, \mathcal{E}^{j}\right\rangle$ is epic for each index $j$, and $B^{u}$ are faithful for $u \in \mathcal{E}$, then the inclusion system $\left\langle\mathcal{I}^{\sharp}, \mathcal{E}^{\sharp}\right\rangle$ defined in Thm. 1 is epic too.

Proof Consider $\langle u, \varphi\rangle:\langle j, \Sigma\rangle \rightarrow\left\langle j^{\prime}, \Sigma^{\prime}\right\rangle$ and $\left\langle u_{1}, \varphi_{1}\right\rangle,\left\langle u_{2}, \varphi_{2}\right\rangle:\left\langle j^{\prime}, \Sigma^{\prime}\right\rangle \rightarrow\left\langle j^{\prime \prime}, \Sigma^{\prime \prime}\right\rangle$ such that

$$
\langle u, \varphi\rangle ;\left\langle u_{1}, \varphi_{1}\right\rangle=\langle u, \varphi\rangle ;\left\langle u_{2}, \varphi_{2}\right\rangle
$$

This means $u ; u_{1}=u ; u_{2}$ and $\varphi ; \varphi_{1} B^{u}=\varphi ; \varphi_{2} B^{u}$. Because $u$ is epi we obtain $u_{1}=u_{2}$. Because $\varphi$ is epi we obtain $\varphi_{1} B^{u}=\varphi_{2} B^{u}$. Because $B^{u}$ is faithful we obtain $\varphi_{1}=\varphi_{2}$. Hence $\left\langle u_{1}, \varphi_{1}\right\rangle=\left\langle u_{2}, \varphi_{2}\right\rangle$.

Corollary 4 The strong inclusion system for many-sorted signature morphisms preserving ad-hoc overloading is epic.

Proof We apply Prop. 2 to the case of Example 9. The standard inclusion system for the category $\mathbb{S e t}$ is epic since surjective functions are epis. The inclusion systems of the categories $B(S)$ are also epic for all sets $S$ because the abstract surjections in $B(S)$ are precisely families of surjective functions. The last condition of Prop. 2 to be checked is that $B^{u}$ are faithful for all surjective functions $u$.

For any surjective function $u: S \rightarrow S^{\prime}$ let us consider $\left(f^{\prime}, g^{\prime}: P^{\prime} \rightarrow P_{1}^{\prime}\right) \in B\left(S^{\prime}\right)$ such that $B^{u}\left(f^{\prime}\right)=B^{u}\left(g^{\prime}\right)$. We have to show that $f^{\prime}=g^{\prime}$. For each $w^{\prime} \in S^{\prime *}$, since $u$ is surjective there exists $w \in S^{*}$ such that $u(w)=w^{\prime}$. Then $f_{w^{\prime}}^{\prime}=f_{u(w)}^{\prime}=B^{u}\left(f^{\prime}\right)_{w}=$ $B^{u}\left(g^{\prime}\right) w=g_{u(w)}^{\prime}=g_{w^{\prime}}^{\prime}$

Corollary 5 For each many-sorted signature $(S, P)$, the strong inclusion system for $(S, P)$ models is epic.

Proof The standard inclusion system for the category $\operatorname{Set}^{S}$ of $S$-sorted sets is epic since each component of an abstract surjection in $\operatorname{Set}{ }^{S}$ is a surjective function. Since $B^{j}=\left\langle\mathcal{I}^{j}, \mathcal{E}^{j}\right\rangle$ are posets, they are trivially epic (as inclusion systems) and $B^{u}$ are trivially faithful for each $u$ index morphism.

Corollary 6 For any $\pi$-institution ( $\mathbb{S i g}$, Sen, $\vdash$ ), if the inclusion system of $\mathbb{S i g}$ is epic, then the corresponding strong inclusion system for theories is epic too.

Proof This follows from the hypothesis that the inclusion system of $\mathbb{S i g}$ is epic, and from the fact that for each $j$ index morphism $B^{j}=\left\langle\mathcal{I}^{j}, \mathcal{E}^{j}\right\rangle$ is trivially epic since it is a poset and consequently for each index morphism $u, B^{u}$ are trivially faithful. 
3.5 The universal property of Grothendieck inclusion systems

In this section we characterize the Grothendieck inclusion system $\left\langle\mathcal{I}^{\sharp}, \mathcal{E}^{\sharp}\right\rangle$ defined by Thm. 1 as a lax colimit enriched by $\mathbb{I} \mathbb{S}$. This refines the characterization of Grothendieck categories as lax colimits [6] and is also consonant to the characterization of Grothendieck institutions as a lax colimit in the 2-category of institutions [6].

Definition 7 (IS-lax colimits) For any pair of functors $F, G:\langle\mathcal{I}, \mathcal{E}\rangle \rightarrow \mathbb{I} \mathbb{S}^{\text {op }}$ (from the underlying category of an inclusion system $\langle\mathcal{I}, \mathcal{E}\rangle$ ), a IIS-lax natural transformation $\mu: F \Rightarrow$ $G$ is a lax natural transformation such that

- for any object $j$ of $\langle\mathcal{I}, \mathcal{E}\rangle$, the functor $\mu^{j}: F(j) \rightarrow G(j)$ is inclusive, and

- for any $u \in \mathcal{I}$, the natural transformation $\mu^{u}$ is abstract inclusion (for the inclusion system of the corresponding functor category; see Example 3).

$\mathbb{I S}$-lax co-cone and $\mathbb{I S}$-lax colimits, respectively, are just lax co-cone and lax colimits, respectively, which are $\mathbb{I S}$-lax as natural transformations.

Proposition 3 For any $\mathcal{E}$-invertible $\mathbb{I S}$-enriched indexed inclusion system $B:\langle\mathcal{I}, \mathcal{E}\rangle \rightarrow$ $\mathbb{I S}{ }^{\text {op }}$, the Grothendieck inclusion system $\left\langle\mathcal{I}^{\sharp}, \mathcal{E}^{\sharp}\right\rangle$ defined by Thm. 1 is the IS-lax co-limit of $B$.

Proof For each index $j$ let the inclusion system $B(j)$ be denoted by $\left\langle\mathcal{I}^{j}, \mathcal{E}^{j}\right\rangle$. We define

- For each index $j$, the functor $\mu^{j}:\left\langle\mathcal{I}^{j}, \mathcal{E}^{j}\right\rangle \rightarrow\left\langle\mathcal{I}^{\sharp}, \mathcal{E}^{\sharp}\right\rangle$ such that

- $\mu^{j}(\Sigma)=\langle j, \Sigma\rangle$ for each object $\Sigma$ of $\left\langle\mathcal{I}^{j}, \mathcal{E}^{j}\right\rangle$, and

- $\mu^{j}(\varphi)=\left\langle 1_{j}, \varphi\right\rangle$ for each arrow $\varphi$ of $\left\langle\mathcal{I}^{j}, \mathcal{E}^{j}\right\rangle$.

Note that $\mu^{j}$ is an inclusive functor since if $\varphi \in \mathcal{I}^{j}$ then of course $\left\langle 1_{j}, \varphi\right\rangle \in \mathcal{I}^{\sharp}$.

- For each index morphism $u: j \rightarrow j^{\prime}$, the natural transformation $\mu^{u}: B^{u} ; \mu^{j} \Rightarrow \mu^{j^{\prime}}$ such that

- $\mu_{\Sigma^{\prime}}^{u}=\left\langle u, 1_{\Sigma^{\prime} B^{u}}\right\rangle$ for each object $\Sigma^{\prime}$ of $\left\langle\mathcal{I}^{j^{\prime}}, \mathcal{E}^{j^{\prime}}\right\rangle$.

Note that $\mu^{u}$ is an abstract inclusion of the functor category $\left\langle\mathcal{I}^{\sharp}, \mathcal{E}^{\sharp}\right\rangle^{\left\langle\mathcal{I}^{j^{\prime}}, \mathcal{E}^{j^{\prime}}\right\rangle}$ if and only if $u \in \mathcal{I}$.

We have thus defined $\mu$ as a IIS-lax co-cone over $B$, with $\left\langle\mathcal{I}^{\sharp}, \mathcal{E}^{\sharp}\right\rangle$ as its vertex.

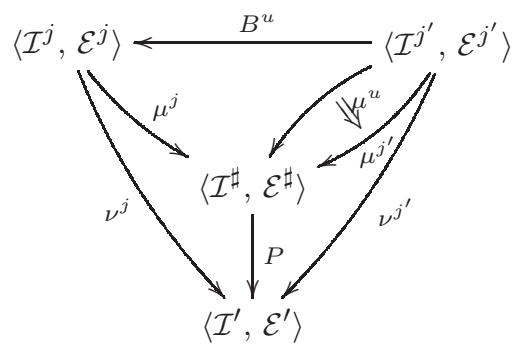

For showing that $\mu$ is a $\mathbb{I S}$-lax co-limit, we consider $\nu$ another $\mathbb{I S}$-lax co-cone over $B$ with an inclusion system $\left\langle\mathcal{I}^{\prime}, \mathcal{E}^{\prime}\right\rangle$ as vertex and prove that there exists an unique inclusive functor $P:\left\langle\mathcal{I}^{\sharp}, \mathcal{E}^{\sharp}\right\rangle \rightarrow\left\langle\mathcal{I}^{\prime}, \mathcal{E}^{\prime}\right\rangle$ such that

$-\mu^{j} ; P=\nu^{j}$ for each index $j$, and

$-\mu^{u} P=\nu^{u}$ for each index morphism $u$. 
These two conditions determine the definition of $P$ as follows:

- for each object $\langle j, \Sigma\rangle$ of $\left\langle\mathcal{I}^{\sharp}, \mathcal{E}^{\sharp}\right\rangle$, we have that $P(\langle j, \Sigma\rangle)=P\left(\mu^{j}(\Sigma)\right)=\nu^{j}(\Sigma)$, and

- for each arrow $\langle u, \varphi\rangle:\langle j, \Sigma\rangle \rightarrow\left\langle j^{\prime}, \Sigma^{\prime}\right\rangle$ of $\left\langle\mathcal{I}^{\sharp}, \mathcal{E}^{\sharp}\right\rangle$, we have that $P(\langle u, \varphi\rangle)=$ $P\left(\left\langle 1_{j}, \varphi\right\rangle ;\left\langle u, 1_{\Sigma^{\prime} B^{u}}\right\rangle\right)=P\left(\left\langle 1_{j}, \varphi\right\rangle\right) ; P\left(\left\langle u, 1_{\Sigma^{\prime} B^{u}}\right\rangle\right)=P\left(\mu^{j}(\varphi)\right) ; P\left(\mu^{u}\left(\Sigma^{\prime}\right)\right)=\nu^{j}(\varphi) ; \nu_{\Sigma^{\prime}}^{u}$.

We leave to the reader the task to calculate that $P$ is a functor.

When $\langle u, \varphi\rangle$ is an abstract inclusion we have that both $u$ and $\varphi$ are abstract inclusions, consequently because $\nu$ is $\mathbb{I S}$-lax natural transformation both $\nu^{j}(\varphi)$ and $\nu_{\Sigma^{\prime}}^{u}$ are abstract inclusions, hence $P(\langle u, \varphi\rangle)=\nu^{j}(\varphi) ; \nu_{\Sigma^{\prime}}^{u}$ is an abstract inclusion. This shows that $P$ is inclusive, which completes the proof of this result.

\subsection{Closed inclusion systems on Grothendieck categories}

The inclusion system construction of Prop. 4 below is much grosser than the Grothendieck inclusion systems of Thm. 1 because it considers a proper inclusion system only for the indices, and not for the fibers. This is the principal reason why this construction is not relevant for multi-logic heterogeneous specifications.

Proposition 4 For any indexed category $B:\langle\mathcal{I}, \mathcal{E}\rangle \rightarrow \mathbb{C} a t^{\text {op }}$ (functor from the underlying category of an inclusion system $\langle\mathcal{I}, \mathcal{E}\rangle$ to the opposite of $\mathbb{C}$ at), its Grothendieck category $B^{\sharp}$ admits an inclusion system such that $\langle u, \varphi\rangle:\langle j, \Sigma\rangle \rightarrow\left\langle j^{\prime}, \Sigma^{\prime}\right\rangle$

- is an abstract inclusion if and only if $u \in \mathcal{I}$ and $\varphi$ is an identity, and

- is an abstract surjection if and only if $u \in \mathcal{E}$.

Proof The facts that abstract inclusions form a poset and that abstract surjections form a sub-category are rather straightforward. Let us now check the unique factorization property. Given any $\langle u, \varphi\rangle:\langle j, \Sigma\rangle \rightarrow\left\langle j^{\prime}, \Sigma^{\prime}\right\rangle$ let $u=e_{u} ; i_{u}$ where $e_{u} \in \mathcal{E}$ and $i_{u} \in \mathcal{I}$. Then $\langle u, \varphi\rangle=\left\langle e_{u}, \varphi\right\rangle ;\left\langle i_{u}, 1_{\Sigma^{\prime} B^{i_{u}}}\right\rangle$ is the unique factorization of $\langle u, \varphi\rangle$ as a composition between an abstract surjection and an abstract inclusion.

Example 12 The closed inclusion systems for many-sorted signatures, models, and theories, respectively, arise as instances of the construction of Prop. 4 for the indexed categories $B$ described in Examples 9, 10, and 11, respectively. Note that in the case of the many-sorted signatures this argument applies to all signature morphisms, including those dthat do not preserve ad-hoc overloading.

Remark 1 In general, the inclusion systems constructed by Prop. 4 do not have unions. This is another reason why the above mentioned construction is irrelevant for multi-logic heterogeneous specifications.

In order to see this failure of unions, let us assume unions for $\langle\mathcal{I}, \mathcal{E}\rangle$ and consider $\left\langle j_{1}, \Sigma_{1}\right\rangle,\left\langle j_{2}, \Sigma_{2}\right\rangle \in \mid B^{\sharp}$. Let $\langle j, \Sigma\rangle=\left\langle j_{1}, \Sigma_{1}\right\rangle \cup\left\langle j_{2}, \Sigma_{2}\right\rangle$. Let $u_{k}: j_{k} \rightarrow j$ denote the corresponding inclusions for $k \in\{1,2\}$. Due to the nature of the abstract inclusions for the Grothendieck category, as defined by Prop. 4, we have that $\Sigma_{k}=\Sigma B^{u_{k}}$ for $k \in\{1,2\}$.

Let us assume $v_{1}$ and $v_{2}$ arrows in the index category such that $v_{1} ; u_{1}=v_{2} ; u_{2}$. Although $v_{1}$ and $v_{2}$ may not exist in general, they do exist in categories which have weak pullbacks, a property which holds in most of the examples of interest, including all examples given in this paper. Then we have that $\Sigma_{1} B^{v_{1}}=\Sigma_{2} B^{v_{2}}$. Since $\Sigma_{1}$ and $\Sigma_{2}$ can be chosen arbitrarily, for reaching a contradiction it is thus enough to chose them in such a way that $\Sigma_{1} B^{v_{1}} \neq \Sigma_{2} B^{v_{2}}$. 


\section{Applications to multi-logic heterogeneous specification}

The heterogeneous specification paradigm involves a system of logics rather than a single underlying logic for a corresponding specification formalism. This is mathematically achieved within the theory of the so-called 'institutions' of Goguen and Burstall [12] by a Grothendieck (flattening) construction which extends the usual Grothendieck construction for categories [15] to institutions [6,20]. A typical example is given by the specification language CafeOBJ whose semantics and design is based upon the Grothendieck construction on the following 'cube' of institutions, with edges representing the sub-logic relationships, formalized by the concept of 'comorphism' between institutions (see [13,8]).

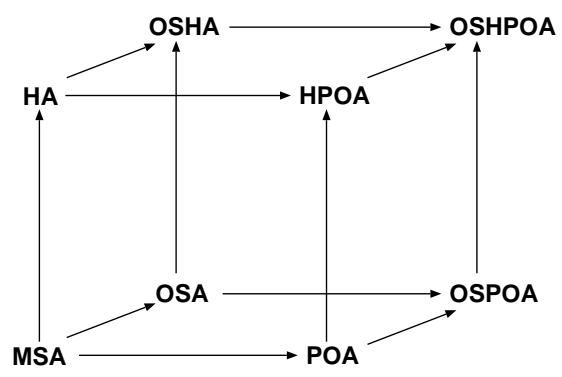

Details on the Grothendieck institution theoretic semantics of CafeOBJ can be found in [9].

Another rather notorious heterogeneous specification framework based upon Grothendieck institutions is that of heterogeneous CASL [22].

The importance of the Grothendieck institution construction on systems of institutions is that by providing a single institution as the underlying mathematical structure for heterogeneous specification frameworks, it is possible to lift important specification theoretic concepts from uniform to heterogeneous frameworks (see [6]). One of these concepts is that of module algebra based upon inclusion systems. This module algebra, or the study of structured specifications, can be done in two variants: a theory oriented one (like in [10] for example), or a more semantic one (like in [24] or [8]). Both of these approaches need a concept of inclusion system for the signatures.

Thus given system of institutions, in fact an indexed institution in the sense of [6],

- such that each institution has an inclusion system for the signatures,

- the signature inclusions being preserved by the comorphisms between the institutions, and

- such that the indexation of the system of institutions comes as a category with an inclusion system $\langle\mathcal{I}, \mathcal{E}\rangle$

the construction of Thm. 1 applied to the resulting enriched indexed inclusion system $B:\langle\mathcal{I}, \mathcal{E}\rangle \rightarrow$ $\mathbb{I} \mathbb{S}^{\text {op }}$ gives the inclusion system for the signatures of the corresponding Grothendieck institution. Because for module algebra the existence of unions is crucial, there is no alternative to this construction since as we have seen above the other possible Grothendieck construction on inclusion systems, that of Prop. 4, does not support unions. Moreover, the construction of Prop. 4 is too gross since it does not consider the 'local' inclusion systems of the fibers.

Often between any two institutions of the system only at most one comorphism is considered, usually representing a 'sub-logic' relationship. As we have already mentioned above, this is also the case of CafeOBJ. This means that $\langle\mathcal{I}, \mathcal{E}\rangle$ is a poset (i.e. $\mathcal{E}$ consists 
only of the identities). On the other hand, the inclusion systems of the categories of signatures of the 'local' institutions involved are not posets in general. This is just the opposite of the situations we have encountered when obtaining the strong inclusion systems for the models and for the theories as Grothendieck inclusion systems (see Examples 10 and 11, respectively).

For such general setup let us look into how the conditions of the general construction of Thm. 1, which gives the construction of the inclusion system for the signatures of the Grothendieck institution, and of Prop. 1, which gives the unions for the above mentioned inclusion system, are satisfied.

1. That the resulting enriched inclusion system $B:\langle\mathcal{I}, \mathcal{E}\rangle \rightarrow \mathbb{I} \mathbb{S}^{\text {op }}$ is $\mathcal{E}$-invertible (as required by Thm. 1) is trivial because $\mathcal{E}$ consists only of identities.

2. However, the existence of unions (Prop. 1) requires that $B$ is invertible. This condition should be checked in each case. Since often the abstract inclusions of $\mathcal{I}$ represent sublogic relationships, in practice this condition is rather easy. In the case of CafeOBJ, for example, the embedding of many-sorted algebra (MSA) into preordered algebra (POA) is an identity on the signatures, while the embedding of MSA into hidden algebra (HA) just forgets the hidden part of the signatures (the left adjoint to this considers MSA signatures as HA signatures with the hidden parts empty).

3. Finally, the existence of unions also requires 'local' unions (which is a property of each institution of the system) and unions at the level of indices. The latter condition means that any two institutions in the system have a least upper bound. When the system of institutions consists only of sub-logic relationships (as in the case of CafeOBJ for example), this means that each two logics of the system should have a common least 'super-logic' in the system.

Acknowledgements The author is grateful to the anonymous referee for careful and competent study of the originally submitted manuscript. His reading has lead to several appropriate suggestions for the paper, including the finding of an error in one of the examples.

\section{Conclusions}

We have extended the Grothendieck flattening from indexed categories to indexed inclusion systems for which the category of the indices comes equipped with an inclusion system too. We have shown that our construction is universal. Besides providing a definitive and complete answer to the problem of inclusion systems for Grothendieck institutions, our general construction subsumes several important 'strong' inclusion systems in use algebraic specification theory and model theory. We have also studied a couple of properties for abstract Grothendieck inclusion systems with special significance for the semantics of heterogeneous multi-logic specification.

It is interesting to note that the inclusion systems of heterogeneous specification formalisms may involve our Grothendieck inclusion system construction at three different levels:

1. at the bottom, the (strong) inclusion systems for many-sorted signatures (for each of the logics/institutions involved),

2. at the mid level, the inclusion system for the signatures of the Grothendieck institution, and

3. at the upper level, the (strong) inclusion system for the theories of the Grothendieck institution. 


\section{References}

1. Marc Aiguier and Fabrice Barbier. An institution-independent proof of the Beth definability theorem. Studia Logica, 85(3):333-359, 2007.

2. Jan Bergstra, Jan Heering, and Paul Klint. Module algebra. Journal of the Association for Computing Machinery, 37(2):335-372, 1990.

3. Francis Borceux. Handbook of Categorical Algebra. Cambridge University Press, 1994.

4. Virgil Emil Căzănescu and Grigore Roşu. Weak inclusion systems. Mathematical Structures in Computer Science, 7(2):195-206, 1997.

5. Răzvan Diaconescu. Extra theory morphisms for institutions: logical semantics for multi-paradigm languages. Applied Categorical Structures, 6(4):427-453, 1998. A preliminary version appeared as JAIST Technical Report IS-RR-97-0032F in 1997.

6. Răzvan Diaconescu. Grothendieck institutions. Applied Categorical Structures, 10(4):383-402, 2002. Preliminary version appeared as IMAR Preprint 2-2000, ISSN 250-3638, February 2000.

7. Răzvan Diaconescu. Elementary diagrams in institutions. Journal of Logic and Computation, 14(5):651674, 2004.

8. Răzvan Diaconescu. Institution-independent Model Theory. Birkhäuser, 2008.

9. Răzvan Diaconescu and Kokichi Futatsugi. Logical foundations of CafeOBJ. Theoretical Computer Science, 285:289-318, 2002.

10. Răzvan Diaconescu, Joseph Goguen, and Petros Stefaneas. Logical support for modularisation. In Gerard Huet and Gordon Plotkin, editors, Logical Environments, pages 83-130. Cambridge, 1993. Proceedings of a Workshop held in Edinburgh, Scotland, May 1991.

11. José L. Fiadeiro and Amilcar Sernadas. Structuring theories on consequence. In Donald Sannella and Andrzej Tarlecki, editors, Recent Trends in Data Type Specification, volume 332 of Lecture Notes in Computer Science, pages 44-72. Springer, 1988.

12. Joseph Goguen and Rod Burstall. Institutions: Abstract model theory for specification and programming. Journal of the Association for Computing Machinery, 39(1):95-146, 1992

13. Joseph Goguen and Grigore Roşu. Institution morphisms. Formal Aspects of Computing, 13:274-307, 2002 .

14. Joseph Goguen and Grigore Roșu. Composing hidden information modules over inclusive institutions. In From Object-Orientation to Formal Methods, volume 2635 of Lecture Notes in Computer Science, pages 96-123. Springer, 2004.

15. Alexandre Grothendieck. Catégories fibrées et descente. In Revêtements étales et groupe fondamental, Séminaire de Géométrie Algébraique du Bois-Marie 1960/61, Exposé VI. Institut des Hautes Études Scientifiques, 1963. Reprinted in Lecture Notes in Mathematics, Volume 224, Springer, 1971, pages 145-94.

16. Robert Harper, Donald Sannella, and Andrzej Tarlecki. Logic representation in LF. In David Pitt, David Rydeheard, Peter Dybjer, Andrew Pitts, and Axel Poigné, editors, Proceedings, Conference on Category Theory and Computer Science, volume 389 of Lecture Notes in Computer Science, pages 250-272. Springer, 1989.

17. Saunders Mac Lane. Categories for the Working Mathematician. Springer, second edition, 1998.

18. Thomas Maibaum, José Fiadeiro, and Martin Sadler. Stepwise program development in $\Pi$-institutions. Technical report, Imperial College, 1990.

19. José Meseguer. General logics. In H.-D. Ebbinghaus et al., editors, Proceedings, Logic Colloquium, 1987, pages 275-329. North-Holland, 1989.

20. Till Mossakowski. Comorphism-based Grothendieck logics. In K. Diks and W. Rytter, editors, Mathematical foundations of computer science, volume 2420 of Lecture Notes in Computer Science, pages 593-604. Springer, 2002.

21. Till Mossakowski. Foundations of heterogeneous specification. In R. Hennicker M. Wirsing, D. Pattinson, editor, Recent Trends in Algebraic Development Techniques, volume 2755 of Lecture Notes in Computer Science, pages 359-375. Springer Verlag, London, 2003.

22. Till Mossakowski. Heterogeneous specification and the heterogeneous tool set. Habilitation thesis, University of Bremen, 2005.

23. Grigore Roşu. Axiomatisability in inclusive equational logic. Mathematical Structures in Computer Science, 12(5):541-563, 2002.

24. Donald Sannella and Andrzej Tarlecki. Specifications in an arbitrary institution. Information and Control, 76:165-210, 1988.

25. Andrzej Tarlecki. Towards heterogeneous specifications. In D. Gabbay and M. van Rijke, editors, Proceedings, International Conference on Frontiers of Combining Systems (FroCoS'98), pages 337-360. Research Studies Press, 2000. 